\title{
Correction: Cosmology at a crossroads
}

Wendy L. Freedman

Nature Astronomy 1, 0121 (2017); published 2 May 2017; corrected 26 May 2017.

In the version of this Comment originally published, Fig. 1 mistakenly included a diagonal black line that has now been removed. 